\title{
Comparison of subcutaneous central venous port via jugular and subclavian access in 347 patients at a single center
}

\author{
BILGIN KADRI ARIBAŞ ${ }^{1}$, KEMAL ARDA $^{1}$, ÖZGE ARIBAŞ ${ }^{2}$, NAZAN ÇILEDAĞ ${ }^{1}$, ZEYNEL YOLOĞLU ${ }^{1}$, \\ ELIF AKTAŞ ${ }^{1}$, TURGUT SEBER ${ }^{1}$, ŞEYHMUS KAVAK ${ }^{1}$, YUSUF COŞAR $^{1}$, HIDIR KAYGUSUZ $^{1}$ and EKREM TEKIN ${ }^{1}$ \\ ${ }^{1}$ Department of Radiology, A. Y. Ankara Oncology Education and Research Hospital, Ankara 06200; \\ ${ }^{2}$ Department of Mathematics, Faculty of Science, University of Ankara, Tandoğan, Ankara 06100, Turkey
}

Received April 20, 2012; Accepted July 26, 2012

DOI: 10.3892/etm.2012.649

\begin{abstract}
The purpose of the present study was to examine whether patency times, including complications of subcutaneous venous chest port insertion using ultrasonography (US) guidance, differ between jugular and subclavian venous access. Between December 2008 and July 2010, subcutaneous venous chest ports were placed in 347 patients by an experienced team. All single-lumen port catheters were placed into jugular and subclavian veins under US and fluoroscopy guidance. Patency times and complication rates of ports via these routes were compared and the variables were age, gender, access, site of malignancy and coagulation parameters. The success of the jugular and subclavian groups was compared by univariate Kaplan-Meier survival analysis and the multivariable Cox regression test. A total of 15 patients underwent port removal due to complications. As a rate per 100 catheter days, ports were explanted in $7(0.0092)$ due to thrombosis, 4 (0.0053) for catheter malposition, one each (0.0013) of port reservoir flip-over, bleeding, port pocket infection, skin necrosis and incision dehiscence, for a total of 15 patients (0.0197). Patency times were not different in the jugular and subclavian veins. Factors were not significant, with the exception of platelet count. There was no significant difference in patency times, including complications, between jugular vein access and subclavian vein access using US. This should be considered when selecting the access method.
\end{abstract}

\section{Introduction}

Infusion therapy via a subcutaneously implanted venous port system is an attractive alternative to infusion via peripheral veins, peripherally inserted central catheters or tunneled catheters (1). The use of subcutaneous infusion ports has become

Correspondence to: Dr Bilgin Kadri Arıbaş, Department of Radiology, A. Y. Ankara Oncology Education and Research Hospital, Menevis sok. 72/11 Asağı Ayran, Ankara 06200, Turkey E-mail: bilginaribas@hotmail.com

Key words: central venous port, interventional radiology, cancer standard practice to obtain long-term venous access for the administration of chemotherapy, antibiotics or parenteral nutrition (2). First surgically implanted by Niederhuber and colleagues in 1982, subcutaneous venous chest ports were subsequently placed by Morris and coworkers with radiological guidance $(3,4)$. Subcutaneous venous chest ports can be placed into jugular and subclavian veins by landmark and radiological methods. Complication rates have been variously reported depending on approach sites and methods (1-22).

To the best of our knowledge, few studies have been published which compare patency periods of port catheters placed into jugular and subclavian veins using radiological techniques (3). Our objective was to examine whether patency times, including complications of subcutaneous venous chest port insertion using ultrasonography (US) guidance, are different via jugular and subclavian venous access.

\section{Patients and methods}

Patients and ports. Between December 2008 and July 2010, subcutaneous venous chest ports were placed under US guidance in 347 patients. From December 2008 to July 2009, our guiding principle was to place the ports via the subclavian vein. We subsequently changed our preference to jugular entry due to the greater ease of this technique. The mean age of the patients was $53.8 \pm 13.9$ years (range, 16-84). Of the patients, $145(41.8 \%)$ were female and $202(58.2 \%)$ were male. All except 1 patient with Behçet's disease had malignancies with or without metastases. The features of the patients and the procedures recorded in this retrospective cohort study were age, gender, access method (jugular or subclavian route), location of primary malignancy, coagulation parameters and complications.

The titanium chambered ports were single-lumen; standard size $(7.2 \mathrm{~F})$ port systems with lock mechanisms for catheter attachment. The port type used was Polysite ${ }^{\circledR}$ (Perouse Laboratoires, Ivry-Le-Temple, France). The ports were usually placed on the right side, but were placed on the left if the right side veins had thromboses. The coagulation parameters were tested prior to each port placement (11) and included platelet count, prothrombin time, international normalized ratio (INR) and activated partial thromboplastin time. An effort was made to correct the deficiencies if any coagulopathy was 
Table I. Demographic features of patients.

\begin{tabular}{|c|c|c|c|}
\hline Characteristics & Total & Jugular group & Subclavian group \\
\hline Age $(\text { years })^{\mathrm{a}}$ & $53.8 \pm 13.9$ & $53.0 \pm 14.0$ & $55.7 \pm 13.7$ \\
\hline \multicolumn{4}{|l|}{ Gender ${ }^{\mathrm{b}}$} \\
\hline Female & $145(41.8)$ & $110(44.4)$ & $35(35.4)$ \\
\hline Male & $202(58.2)$ & $138(55.6)$ & $64(64.6)$ \\
\hline Access vein ${ }^{b}$ & $347(100)$ & $248(71.5)$ & $99(28.5)$ \\
\hline Platelet count $/ \mathrm{nl}^{\mathrm{a}}$ & $307.9 \pm 120.2$ & $306.9 \pm 123.9$ & $310.4 \pm 110.8$ \\
\hline Prothrombin time $(\mathrm{sec})^{\mathrm{a}}$ & $11.8 \pm 1.5$ & $11.4 \pm 1.2$ & $12.8 \pm 1.7$ \\
\hline International normalized ratio (INR) ${ }^{\mathrm{a}}$ & $0.980 \pm 0.137$ & $0.946 \pm 0.111$ & $1.068 \pm 0.157$ \\
\hline Activated partial thromboplastin time $(\mathrm{sec})^{\mathrm{a}}$ & $28.2 \pm 4.5$ & $28.1 \pm 4.5$ & $28.3 \pm 4.7$ \\
\hline \multicolumn{4}{|l|}{ Localization of primary malignancies ${ }^{b}$} \\
\hline Head-neck & $62(17.9)$ & $45(18.2)$ & $17(17.2)$ \\
\hline Breast-thorax & $29(8.4)$ & 21 & $8(8.1)$ \\
\hline Abdominopelvic & $237(68.3)$ & $170(68.8)$ & $67(67.7)$ \\
\hline Extremity-other ${ }^{c}$ & $18(5.2)$ & $11(4.5)$ & $7(7.1)$ \\
\hline \multicolumn{4}{|l|}{ Mortality } \\
\hline Positive & $20 \quad(5.8)$ & $12(4.8)$ & $8(8.1)$ \\
\hline Negative & $327(94.2)$ & $236(95.2)$ & $91(91.9)$ \\
\hline Patency periods (days) ${ }^{\mathrm{d}}$ & $219.5 \pm 145.0(1-550)$ & $199.2 \pm 122.5(1-471)$ & $270.4 \pm 180.9(1-550)$ \\
\hline
\end{tabular}

detected. Prophylactic antibiotics were not administered to any patient, including those with fever, until positive culture results. Exclusion criteria were active systemic or local infections, coagulopathy (defined as platelet count $<50 / \mathrm{nl}$ and/or prothrombin time $>18 \mathrm{sec}$ and/or INR $>1.5$ ) and the inability to provide informed consent (23).

Technique of the procedure. Two of the skilled interventional radiologists had 21 years of experience in interventional radiology, including venous catheterization. The other 6 radiologists performed the procedure under their observation. The single-lumen port catheters were placed into the jugular and subclavian veins under US and fluoroscopy guidance in an interventional radiology suite. Patients were placed in the supine position. The pectoral and neck regions were cleansed with povidone-iodine twice. Preprocedural sedation was not administered, except to uncooperative patients. Sterile technique was used, in which the skin at the insertion site was extensively cleansed on the neck or chest. We performed in full surgical scrubs, wearing surgical caps and masks. US guidance (Famio 8; Toshiba, Japan) was performed in all procedures. A high-resolution (11 MHz) linear-array transducer was used with standard accords in all procedures as B-gain, $80 \mathrm{~dB}$; dynamic range, 55; frame per sec (fps), 15 and standard depth, $5.3 \mathrm{~cm}$. Sterile US gel and sterile drapes were used to cover the US probe and cable. A skin incision of $1 \mathrm{~cm}$ was made over the jugular or subclavian vein site following local anesthesia administration. Principally, the subclavian vein was punctured in the mid or lateral third of the clavicle to avoid pinch-off. In all patients, venous entry was performed with an $18 \mathrm{G}$
Seldinger needle and the tip of the guide-wire was advanced into the vena cava. After puncture, the subcutaneous pocket was dissected. Following local anesthesia with $2 \%$ prilocaine (Citanest $^{\circledR}$; Eczacıbaşı, Turkey), a 2.5- to 3-cm incision was made $\sim 3 \mathrm{~cm}$ caudal to the clavicle with a number 15 bistoury. The pocket site was the same for jugular and subclavian access. Using sharp and blunt dissection, a pocket was created under the fascia of the pectoral muscle and caudally dissected with a clamp and finger. A sponge was placed into the pocket for hemostasis prior to port placement. The catheter connected to the port chamber was tunneled with a trochar to the venous entry site. The catheter was flushed with diluted heparin solution using a Huber needle. The Huber needle remained in the port chamber to prevent flip-over until the end of the procedure. Stay sutures for the port base were not used in any patient, even if the patient had loose subcutaneous tissue. The port catheter was advanced through the peel-away sheath into the vena cava-right atrium junction. After insertion, the catheter tip was evaluated using fluoroscopy. Port efficacy was checked with an aspiration of blood, and the reservoir was flushed with heparinized saline solution $(9 \mathrm{cc} 0.9 \% \mathrm{NaCl}$ plus $1 \mathrm{cc}$ heparin) to show any leakage. The incision was closed with resorbable 3-0 vicryl subcutaneous sutures. The skin of the pocket and venous puncture site was closed with 2.0 silk sutures. A digital lung graph was used at the completion of the procedure to evaluate for pneumothorax, catheter kinking and catheter tip position in accordance with the guidelines (23). The port was used for treatment $3 \mathrm{~h}$ after the procedure. Port catheters were flushed with heparinized saline solution after each use and, thereafter, monthly if not used. 
Table II. Complications leading to port removal.

\begin{tabular}{|c|c|c|c|c|c|c|c|}
\hline \multirow[b]{2}{*}{ Complication } & \multirow[b]{2}{*}{$\mathrm{n}(\%)^{\mathrm{a}}$} & \multicolumn{2}{|c|}{ Jugular } & \multicolumn{2}{|c|}{ Subclavian } & \multirow[b]{2}{*}{ Term (days) } & \multirow[b]{2}{*}{ Rate/100 catheter days } \\
\hline & & $\mathrm{n}(\%)$ & P-value & $\mathrm{n}(\%)$ & P-value & & \\
\hline Thrombosis ${ }^{\mathrm{b}}$ & $7(2.0)$ & $5(2.0)$ & 0.0101 & $2(2.0)$ & 0.0074 & 375 & 0.0092 \\
\hline Malposition & $4(1.2)$ & $1(0.4)$ & 0.0020 & $3(3.0)$ & 0.0112 & 833 & 0.0053 \\
\hline Flip-over & $1(0.3)$ & $1(0.4)$ & 0.0020 & 0 & 0 & 312 & 0.0013 \\
\hline Hemorrhage & $1(0.3)$ & $1(0.4)$ & 0.0020 & 0 & 0 & 1 & 0.0013 \\
\hline Infection $^{c}$ & $2(0.6)$ & $1(0.4)$ & 0.0020 & $1(1.0)$ & 0.0037 & 142 & 0.0026 \\
\hline Skin necrosis ${ }^{\mathrm{c}}$ & $1(0.3)$ & $1(0.4)$ & 0.0020 & 0 & 0 & 132 & 0.0013 \\
\hline Total $^{\mathrm{d}}$ & $16(4.4)$ & $10(4.0)$ & 0.0182 & $6(6.1)$ & 0.0224 & 1,795 & 0.0197 \\
\hline
\end{tabular}

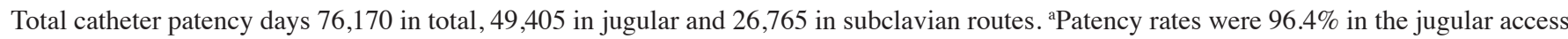
and $93.9 \%$ in subclavian access groups. ${ }^{b}$ Five $(71.4 \%)$ female patients and $2(28.6 \%)$ male patients. ${ }^{c}$ Infection accompanied by skin necrosis in 1 patient. ${ }^{\mathrm{d}}$ Sixteen complications were observed in 15 patients.

Follow-up, analysis of results and statistical analysis. Informed consent was obtained from each patient at the time of the intervention, in accordance with the Helsinki Declaration. All data were obtained from our files and the hospital electronic database system after receiving the permission of the Institutional Review Board (IRB). The follow-up term was from placement until removal of the port, the last follow-up of the patient or mortality date. Patients were censored due to mortality and the last follow-up. Groups were divided by entry methods, which were the jugular and subclavian access groups. Age, gender, access, site of malignancy and coagulation parameters were the variables in the multivariable analysis. The sites of primary malignancies were divided into four regions, which were head-neck, breast-thorax, abdominopelvic and extremity involving $>1$ region. These regions were grouped together since they were individually small in number.

Complications were defined according to the guidelines of the Society of the Interventional Radiology Standards of Practice Committee (5). Major complications result in admission to a hospital for therapy (for outpatient procedures), an unplanned increase in the level of care, prolonged hospitalization, permanent adverse sequelae or mortality (5). Complications were divided into early (<30 days) and late (>30 days) as in the reporting standards (23).

Event (failure) was defined as unplanned port removal due to complications, so the groups were divided into failure and success. Patency times of the ports via these routes were compared using univariate Kaplan-Meier survival analysis and the multivariable Cox regression test. $\mathrm{P}<0.05$ and $95 \%$ confidence interval (CI) in the analysis were considered to indicate a statistically significant difference. Survival plots for catheter patency were obtained from the statistical analysis. Statistical analysis was performed by a mathematician (Ö.A.).

\section{Results}

Nine patients, of which 1 was in the subclavian group, had abnormal bleeding parameters; these were corrected before their ports were implanted. No major complications were detected during the procedure. Two major complications occurred following the procedure in the jugular group; a late complication after 132 days in a 69 -year-old male patient, who experienced infection by skin necrosis, and an early complication on the second day, which was bleeding due to prolonged INR (1.70) in a 29-year-old male patient to whom blood was administered before the port was removed. Patient features and patency periods of subclavian and jugular catheters are shown in Table I. Port catheters were placed into jugular and subclavian veins in 248 (71.5\%) and 99 patients $(28.5 \%)$, respectively. The mean number of catheter days was larger in the subclavian than in the jugular access groups, 270.4 vs. 199.2 days. Ports were placed on the left side in 4 patients; jugular in 2 and subclavian in the remaining 2 patients.

Complications without port removal were observed in $4.9 \%$ of patients (17 patients). These included erythema and itching in 2 patients, opening of sutures in 3 patients and bleeding in 12 patients. Ports were explanted due to the end of treatment in 5 patients and 20 mortalities were observed during follow-up, which were censored. Port removal due to complications was observed in 15 patients, 9 in the jugular group and 6 in the subclavian group (Table II).

Thrombosis occurred in 2 patients, malposition in 3 (Fig. 1) and infection in 1 patient when subclavian access was used, whereas there was thrombosis in 5 patients $(3$ catheter thromboses, 1 jugular and the other brachiocephalic vein thrombosis) and 1 case each of malposition, bleeding, reservoir flip-over and skin necrosis with wound infection in the jugular access group. Of the complications, 6 were early (4 in the jugular and 2 in the subclavian group) and 9 were late (5 in the jugular and 4 in the subclavian group).

The port removal rate due to complication per 100 port catheter days was 0.00182 in the jugular and 0.00224 in the subclavian entry groups. Also, the port infection rate per 100 port catheter days regarding the procedure was 0.0020 in the jugular vs. 0.0037 in the subclavian group.

The graph in Fig. 2 shows the cumulative survival for catheter patency times of the jugular and subclavian access groups in the Kaplan-Meier survival analysis. Log-rank test 

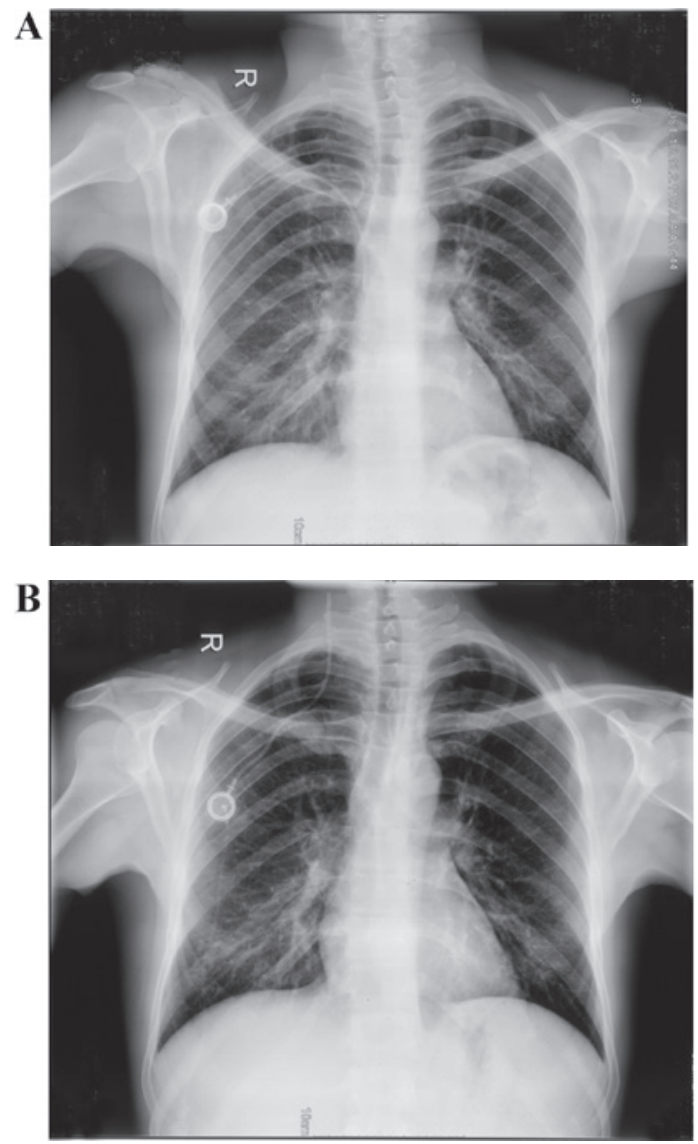

Figure 1. A 36-year-old male patient with colon carcinoma. (A) Upright posteroanterior chest radiograph showing that a right subclavian port was implanted into the chest wall and that the catheter was placed into the lower half of the vena cava just after the procedure. (B) Malposition due to dislocation of this port catheter can be observed in the right jugular vein. This developed 3 months later. The catheter was subsequently placed back into the vena cava via jugular access.

did not detect any significant differences between the groups $(\mathrm{P}=0.662)$.

The graph in Fig. 3 shows the total cumulative survival for mean catheter patency time by the multivariable Cox regression test. Age $(\mathrm{P}=0.252)$, gender $(\mathrm{P}=0.775)$, access vein groups $(\mathrm{P}=0.369)$, site of primary malignancy $(\mathrm{P}=0.607)$ and coagulation parameters, with the exception of platelet count $(\mathrm{P}=0.043)$, were not significant variables in this multivariable test. Coagulation parameters were platelet count $(\mathrm{P}=0.043)$, prothrombin time $(\mathrm{P}=0.526)$, INR $(\mathrm{P}=0.289)$ and activated partial thromboplastin time $(\mathrm{P}=0.087)$ in the multivariable test. Their platelet counts $(/ \mathrm{nl})$ were $275.5 \pm 96.8$ in the failure group and $309.4 \pm 121.0$ in the successful group.

\section{Discussion}

Image-guided insertion of subcutaneous chest ports has a number of advantages compared with unguided insertion, resulting in a higher success rate and fewer complications (6). The major difference between the techniques is the use of fluoroscopy and US $(7,8)$. US guidance reduces the number of mechanical complications, the number of catheter placement failures and the time required for insertion. However, US guidance use during subclavian venous catheterization has

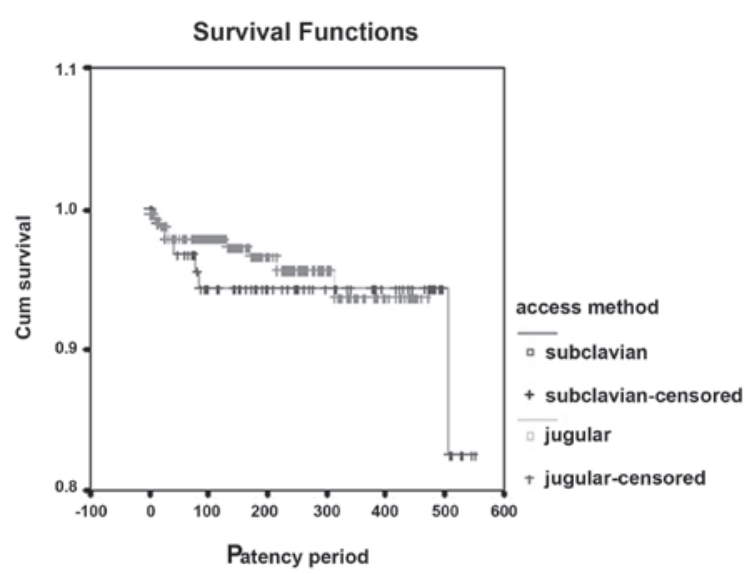

Figure 2. Graph of Kaplan-Meier survival test showing the cumulative catheter patency times of the jugular and subclavian vein access groups with no significant differences.

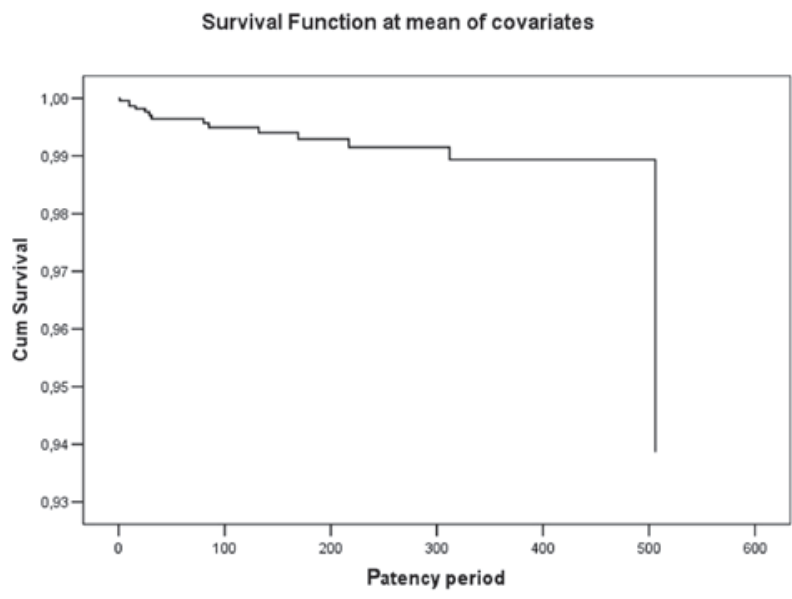

Figure 3. Graph of Cox multivariable regression test showing mean catheter patency time. Confidence interval (CI) for exponent B was $95 \%$, entry $5 \%$ and removal $10 \%$, maximum iterations 20 , model entry and model display information were selected at each step.

had mixed results in clinical trials, probably due to anatomical reasons (9).

Our complication rate was low due to the use of imaging guidance, compared with the complication rates of the landmark method, as reported in the literature. The use of two-dimensional ultrasound (2D-US) guidance during internal jugular catheterization has been demonstrated to lead to a reduction in the rates of unsuccessful cannulation, carotid artery puncture and hematoma formation when compared with the anatomical landmark technique (10-13).

In the literature, the most common complications after implantation were thrombosis, catheter dysfunction and infections (2). Catheter-related thrombosis is one of the most significant complications; its frequency ranges from 0.67 to $5 \%$ (2). Our thrombosis rate was within this range at $2 \%$ (0.0092 per 100 catheter days) and was slightly higher in the jugular access group. However, it is debatable whether Plumhans et al (3) observed vein thrombosis in 3\% of the subclavian group and in $1 \%$ of the jugular group when thrombosis was reported in $1 \%$ of subclavian ports (8) and in $1.7 \%$ of jugular placements (6) in other studies. 
Female patients and patients with lung cancer also had an elevated risk of developing a thrombosis (2). We detected more frequent thrombosis in female patients ( $71.4 \%$ compared with $28.6 \%$ in male patients) but this was not significant in multivariable analysis. Also, we were unable to differentiate thrombosis detection between patients with various malignancy localizations.

The risk of catheter-related infection was reportedly lower for subclavian vein access than for jugular or other access sites; however, no randomized trial has satisfactorily compared infection rates for catheters placed in jugular, subclavian and femoral sites $(9,11,13-15)$. We could neither find a difference in the infection rates $(0.0020$ in jugular vs. 0.0037 in subclavian per 100 catheter days) nor incubate the responsible microorganism in cultures of the two port site infections due to the antibiotic treatments administered.

Catheter-related complications also include necrosis of the skin, malpositioning of the catheter tip, dislocation, embolization, rupture and compression of the catheter, although these are rare (2). Catheter tip position is less susceptible to migration when placed through the internal jugular vein (3). Accordingly, we detected this in the present study, and our catheter malposition rate per 100 catheter days was 0.0020 in the jugular vs. 0.0112 in the subclavian route. Skin necrosis may be observed in port placement $(2,4)$. Skin erosion has been reported in 0 to $1 \%$ of cases in the literature $(1,4)$, and our rate was $0.3 \%$ ( 0.0013 per 100 catheter days).

Subclavian venipuncture has been the most popular route for transition and long-term central venous cannulation, although perioperative complications occur in up to $12 \%$ of the patients (11). Currently, radiologists prefer the internal jugular vein since it is makes catheterization easier $(3,4,6,13)$. The main advantages of jugular versus subclavian access are the reduced periprocedural complications, better ultrasound control, no pinch-off and lower migration and venous stenosis rate $(3,10,16)$. Also, Plumhans et al (3) reported that their results demonstrated an approximately 50\% reduction of pain perception when the port-catheter was introduced via the internal jugular vein. Conversely, Lorch et al (1) preferred access through the subclavian vein since the distance to the vena cava and right atrium is short so no tunneling is necessary, thus shortening the procedure time; it also requires no second incision at the neck, which may be an advantage, especially in cachectic patients.

When possible, a lateral puncture of the subclavian vein should be performed if subclavian access is chosen to avoid pinch-off $(1,14,15)$. Fluoroscopic and/or ultrasound-guided access to the subclavian vein also prevents catheter buckling or breakage due to 'pinching' between the first rib and the clavicle (17). The success rate of the technique was higher in subclavian access in 55 patients for Brooks et al (18) with US guidance. Also, we did not observe visible catheter pinching, but some pinch-off may have an effect on catheter thrombosis in subclavian entry.

Previously, two studies $(14,15)$ revealed that port placement via the subclavian vein was as successful as studies performed via the jugular vein reported in the literature. Biffi et al (7) prospectively compared subclavian and jugular port placements using the radiological and landmark methods and found no differences in success. Furthermore, we did not find any significant differences between catheter patency periods of subclavian and jugular port placements, including complications, using the same method.

Port inversion (turning over inside the port's fibrous capsule) is an extremely rare complication $(19,20)$. Postulated risk factors for port inversion include loose or redundant subcutaneous tissues and large pocket size (19). Thus, fixing the port chamber in the subcutaneous tissue with sutures is not necessary if the port pocket size is adequate (1). Also, certain studies have, as we observed in the present study, demonstrated that starting chemotherapy on the day of port catheter implantation is safe, and does not increase the frequency of acute or chronic complications (21).

This study has some restrictions. Among them, the number of the patients was limited due to single center experience. Also, the patients were not equally distributed between the two access groups whereby the mean follow-up period of the subclavian group, whose success would be expected at a lower rate, was longer than that of the jugular group.

In our presented data, the periprocedural complication rate was $0 \%$. We would like to emphasize that skilled interventional staff performed the procedure. Our experience has an important role in the absence of periprocedural complication, especially in subclavian entry. Thus, our choice of entry method had a minimal effect on technique success.

We did not find any factor to be effective on catheter patency times by multivariable analysis, with the exception of platelet count. However, the difference in platelet counts was approximately $\mathrm{P}=0.05$. This may be due to the limitations of our study. This may be examined in further studies.

The rate of symptomatic thrombosis in subclavian access was higher in the study by Trerotola et al (22). However, US markedly decreased failure and complication rates in subclavian entry in the study by Brooks et al (18), which was also true in our study. Data extracted from the current study may increase the amount of evidence in the literature for the positive effect of US in subclavian entry.

In conclusion, there was no significant difference in patency times, including complications, between jugular vein and subclavian vein access when using US. This should be considered when selecting the access method.

\section{References}

1. Lorch H, Zwaan M, Kagel C and Weiss HD: Central venous access ports placed by interventional radiologists: experience with 125 consecutive patients. Cardiovasc Intervent Radiol 24: 180-184, 2001.

2. Caers J, Fontaine C, Vinh-Hung V, et al: Catheter tip position as a risk factor for thrombosis associated with the use of subcutaneous infusion ports. Support Care Cancer 13: 325-331, 2005.

3. Plumhans C, Mahnken AH, Ocklenburg C, et al: Jugular versus subclavian totally implantable access ports: catheter position, complications and intrainterventional pain perception. Eur J Radiol 79: 338-342, 2011.

4. Cil BE, Canyiğit M, Peynircioğlu B, et al: Subcutaneous venous port implantation in adult patients: a single center experience. Diagn Interv Radiol 12: 93-98, 2006.

5. Lewis CA, Allen TE, Burke DR, et al: Society of Interventional Radiology Standards of Practice Committee: Quality improvement guidelines for central venous access. J Vasc Interv Radiol 14: S231-S235, 2003.

6. Yip D and Funaki B: Subcutaneous chest ports via the internal jugular vein. A retrospective study of 117 oncology patients. Acta Radiol 43: 371-375, 2002. 
7. Biffi R,CorradoF, de Braud F, et al:Long-term,totally implantable central venous access ports connected to a Groshong catheter for chemotherapy of solid tumours: experience from 178 cases using a single type of device. Eur J Cancer 33: 1190-1194, 1997.

8. Funaki B,Szymski GX, Hackworth CA, et al: Radiologic placement of subcutaneous infusion chest ports for long-term central venous access. AJR Am J Roentgenol 169: 1431-1434, 1997.

9. McGee DC and Gould MK: Preventing complications of central venous catheterization. N Engl J Med 348: 1123-1133, 2003.

10. Hind D, Calvert N, McWilliams R, et al: Ultrasonic locating devices for central venous cannulation: meta-analysis. BMJ 327 361, 2003.

11. Biffi R, Orsi F, Pozzi S, et al: Best choice of central venous insertion site for the prevention of catheter-related complications in adult patients who need cancer therapy: a randomized trial. Ann Oncol 20: 935-940, 2009.

12. Di Carlo I, Pulvirenti E, Mannino M and Toro A: Increased use of percutaneous technique for totally implantable venous access devices. Is it real progress? A 27-year comprehensive review on early complications. Ann Surg Oncol 17: 1649-1656, 2010.

13. Karakitsos D, Labropoulos N, De Groot E, et al: Real-time ultrasound-guided catheterisation of the internal jugular vein: a prospective comparison with the landmark technique in critical care patients. Crit Care 10: R162, 2006.

14. Akahane A, Sone M, Ehara S, Kato K, Tanaka R and Nakasato T: Subclavian vein versus arm vein for totally implantable central venous port for patients with head and neck cancer: a retrospective comparative analysis. Cardiovasc Intervent Radiol 34 1222-1229, 2011.

15. Sakamoto N, Arai Y, Takeuchi Y, Takahashi M, Tsurusaki M and Sugimura K: Ultrasound-guided radiological placement of central venous port via the subclavian vein: a retrospective analysis of 500 cases at a single institute. Cardiovasc Intervent Radiol 33: 989-994, 2010.
16. Teichgräber UK, Kausche S, Nagel SN and Gebauer B: Outcome analysis in 3,160 implantations of radiologically guided placements of totally implantable central venous port systems. Eur Radiol 21: 1224-1232, 2011.

17. Shetty PC, Mody MK, Kastan DJ, Sharma RP, Burke MW and Venugopal C: Outcome of 350 implanted chest ports placed by interventional radiologists. J Vasc Interv Radiol 8: 991-995, 1997.

18. Brooks AJ, Alfredson M, Pettigrew B and Morris DL: Ultrasound-guided insertion of subclavian venous access ports. Ann R Coll Surg Engl 87: 25-27, 2005.

19. McNulty NJ, Perrich KD, Silas AM, Linville RM and Forauer AR: Implantable subcutaneous venous access devices: is port fixation necessary? A review of 534 cases. Cardiovasc Intervent Radiol 33: 751-755, 2010.

20. de Costa BR, Dickey K and Greenwood L: A practical approach for repositioning flipped venous access ports. J Vasc Interv Radiol 11: 213-214, 2000

21. Ozdemir NY, Abali H, Oksuzoğlu B, Budakoglu B, Akmangit I and Zengin N: It appears to be safe to start chemotherapy on the day of implantation through subcutaneous venous port catheters in inpatient setting. Support Care Cancer 17: 399-403, 2009.

22. Trerotola SO, Kuhn-Fulton J, Johnson MS, Shah H, Ambrosius WT and Kneebone PH: Tunneled infusion catheters: increased incidence of symptomatic venous thrombosis after subclavian versus internal jugular venous access. Radiology 217: 89-93, 2000.

23. Silberzweig JE, Sacks D, Khorsandi AS and Bakal CW; Society of Interventional Radiology Technology Assessment Committee: Reporting standards for central venous access. J Vasc Interv Radiol 14: S443-S452, 2003. 